\title{
Impact of marital status during diagnosis on cancer-caused specific survival in acute myeloid leukemia patients: a case- control and population-based study
}

\author{
Zhuojun Zheng ${ }^{1,2,3,4, *}$, Yuandong Zhu ${ }^{1}$, Xiaodong $\mathbf{L i}^{2,3,4,5, *}$, Wenwei $\mathrm{Hu}^{2,3,4,5}$ and \\ Jingting Jiang ${ }^{2,3,4}$ \\ ${ }^{1}$ Department of Hematology, The Third Affiliated Hospital of Soochow University, Changzhou, China \\ ${ }^{2}$ Department of Tumor Biological Treatment, The Third Affiliated Hospital of Soochow University, Changzhou, China \\ ${ }^{3}$ Cancer Immunotherapy Engineering Research Center of Jiangsu Province, Changzhou, China \\ ${ }^{4}$ Institute of Cell Therapy Soochow University, Changzhou, China \\ ${ }^{5}$ Department of Oncology, The Third Affiliated Hospital of Soochow University, Changzhou, China \\ ${ }^{*}$ Co-first authors of this work
}

Correspondence to: Jingting Jiang, email: jiangjingting@suda.edu.cn

Wenwei Hu, email: huwenwei1979@163.com

Keywords: acute myeloid leukemia, marital status, SEER, survival analysis, subgroup analysis

Received: November 24, $2016 \quad$ Accepted: February 28, $2017 \quad$ Published: April 09, 2017

Copyright: Zheng et al. This is an open-access article distributed under the terms of the Creative Commons Attribution License 3.0 (CC BY

3.0), which permits unrestricted use, distribution, and reproduction in any medium, provided the original author and source are credited.

\section{ABSTRACT}

Objective: This study investigated the impact of marital status on cancer-caused specific mortality among acute myeloid leukemia (AML) patients in the United States. Methods: We used the Surveillance, Epidemiology and End Results program to identify 50,825 patients who had their clinical and follow-up information available and were diagnosed for AML between the years 1988 and 2015. The univariate and multivariable Cox regression models were used to analyze the patient data, and to minimize the group differences due to covariates between groups, a 1:1 propensity score matching was used in subsequent subgroup analysis. Results: Our study demonstrated that married patients were less likely to die due to AML after adjusting for demographic and clinicopathological variables, than patients with variable unmarried status. Further analysis indicated that widowed, divorced and never married status correlated with poor cancer-cause specific survival than being married in almost all subgroups after being adjusted for the aforementioned variables $(P<0.05)$. However, the difference between married and separated was not apparent. Moreover, similar survival analysis results were also observed in the 1:1 matched subgroups of marital status, but they displayed varied prognostic factors between them. The association of survival benefit with marriage in AML was consistent with the published survival benefit of conventional therapeutic approaches. Conclusion: Overall, our study concluded that unmarried AML patients were at greater risk of cancer-specific mortality than married, and thus indicated that physicians should focus on health care strategies that target social support, in order to reduce the cancer-specific mortality in unmarried patients.

\section{INTRODUCTION}

Among the hematological malignancies, humans usually display high frequency of acute leukemia. In adults, acute myeloid leukemia (AML) is more common than acute lymphoblastic leukemia, and its incidence gradually increases with age and rate is about 16.2 cases per 100,000 individuals with age over 65 year. The underlying mechanism of AML is still unknown, and the only significant therapeutic advantage achieved in the 
last decade has been the long-term cure of patients with acute promyelocytic leukemia (APL) while invasion is absence in advance of non-APL. Currently, the 5-year overall survival rate is between 0 to $40 \%$, and complete remission rate is $\geq 80 \%$ in younger patients, while it is disappointingly very low in older patients [1].

The social support among the many factors actually exerts a significant effect on the clinical outcome, especially in patients with malignant diseases [2, 3]. It is well known that marital status has been the most important social support, which also associates with a variety of other important social factors [4]. Married patients generally show better recovery from a single malignancy, as they seem to receive more social support, including practical support and financial resources. Some studies have demonstrated that marital status is an independent prognostic factor in multiple cancers [5-8], and the survival benefits due to marriage has been greater than the published survival benefits from chemotherapy in several cancers [9-12]. In addition, marital status linkage with delayed diagnosis, lack of treatment and social support, leads to poor survival $[5,13,14]$. There has been conflicting studies about the effect of marriage on acute leukemia. For instance, the study by Borate et. al. showed that single (never married) and divorce status were poor prognostic factors for survival in younger AML patients [15]. However, on the contrary, the study by Fintel et. al. [16] reported that marriage did not have any influence on the survival outcome in adolescent and young adults with AML, thereby suggesting that social issues like marital status were less important than disease-specific therapies. Thus, we believe that a better understanding of the impact of marital status on AML would lead to better understanding of the importance of social mechanisms in the management of this malignancy and can help to establish a more holistic approach to improve patient outcomes. Hence, we in this study have tried to explore the underlying mechanisms of the correlation between marital status and the survival of younger and elderly AML patients.

\section{MATERIALS AND METHODS}

\section{Data source}

This study used Surveillance, Epidemiology, and End Results (SEER) database released in November 2015 as a data source. It included data from 18 population-based registries from 1973 to 2013 and covers approximately $30 \%$ of the US population. The SEER program registries routinely collected data on patient demographics, primary tumor site, tumor morphology and stage at diagnosis, first course of treatment, and follow-up for vital status. The mortality data reported by SEER were updated annually by the National Center for Health Statistics [17]. The National Cancer Institute's SEER*Stat software (Surveillance Research Program, National Cancer Institute SEER*Stat software, www.seer.cancer.gov/seerstat) (Version 8.3.2) was used to collect all the necessary information.

\section{Inclusion criteria}

To identify appropriate patients for this study, the following inclusion criteria was used: a) Patients should be diagnosed with AML (International Classification of Diseases for Oncology, Third Edition [ICD-O-3], codes 9840/3, 9861/3, 9865/3, 9866/3, 9867/3, 9869/3, 9871/3, $9872 / 3,9873 / 3,9874 / 3,9895 / 3,9896 / 3,9897 / 3,9898 / 3$, $9910 / 3,9911 / 3,9920 / 3$ ), between the year 1988 and 2013, and were aged 16 or older at the time of diagnosis. AML, NOS; refered to acute myeloid leukemia with FAB or WHO type, not otherwise specified, included acute nonlymphocytic leukemia, acute granulocytic leukemia, acute myelogenous leukemia and acute myelocytic leukemia according to ICD-O-3. b) Patients who had been histologically confirmed, diagnosed and were actively followed up. However, the patients were excluded if they were younger than 16 years; had insufficient or unknown clinicopathologic-profile; had unknown marital status, cause of death or survival information. Finally, 50825 patients were included for analysis in our study.

\section{Study variables}

The following variables were extracted from the SEER database, including marital status, sex, race, age at diagnosis, AML subtype, cause-specific survival (CSS), and adjuvant therapy. Marital status at diagnosis referred to "the status at diagnosis" when not otherwise specified (NOS), and was categorized as married, divorced, widowed, separated and single (never married), and also categorized as a binary variable into married and unmarried (including single, divorced or separated, and widowed groups) in matched case-control analysis. Race was classified into African American, non-Hispanic white, and others (American Indian/AK Native, Asian/ Pacific Islander) as provided by the SEER database. Age at diagnosis was divided into different groups: 16 to 35 year, 36 to 55 year, 56 to 75 year, 76 to 95 year, and 96 year or over. Data of AML subtype were all coded according to ICD-O-3. Age at the diagnosis and AML subtype were categorized as a binary variable into 16 to 55 year vs. 56 year or over and AML, NOS vs. others in matched casecontrol analysis. Adjuvant therapy was categorized as none radiotherapy, beam radiation or radioisotopes, and radiotherapy unknown. 


\section{Outcome measurement}

Previous studies have reported about overall survival (OS), while cancer-caused specific survival (CSS) was neglected. CSS usually provides more accurate information than OS about the death caused by primary cancer. Thus, we in our study only focussed on CSS as the primary outcome, and it was defined from the date of diagnosis to the date of cancer-specific death and was shown as "SEER cause-specific survival". Deaths attributed to AML were treated as events. Patients who died from other causes or were still alive at the time of the last follow-up were treated as censored observations.

\section{Statistical analysis}

Clinicopathological baseline characteristics were compared with Pearson chi-square test for categorical data. CSS rate was calculated by Kaplan-Meier curve, and compared by log-rank (Mantel-Cox) test. Univariate and multivariate Cox proportional hazard models were built to determine survival outcome and risk factors. To minimize the group differences on covariates between patients who were married, unmarried or never married, a 1:1 propensity score matching was used. Propensity scores were calculated with logistic regression, with multiple imputation and backward elimination with a significance level of 0.05 . Matching on the propensity scores was done with a nearest-neighbor algorithm, allowing a maximum tolerated difference between propensity scores of no larger than 0.1 of the propensity score standard deviation [18]. Group differences were estimated by cross table chi-square test. The log-rank test (Kaplan-Meier curve) was applied to estimate median cancer-CSS between matched groups. For this study, data points about sex, race, AML subtype, age at diagnosis and adjuvant therapy were included in propensity matching. The respective matching ratios of 1:1 were selected to maximize the number of matched pairs without exceeding the maximum tolerated difference between matched propensity scores. All statistical analyses were performed using the Statistical Package for the Social Sciences (SPSS) software version 22 (SPSS Inc., Chicago, IL, USA). The $P$ value of $<0.05$ represented statistically significant difference.

\section{RESULTS}

\section{Demographic and clinicopathological baseline characteristics}

Based on the inclusion criteria, a total of 50,825 eligible patients were identified, including 27,510 male and 23,315 female patients. Among these, 30,006 were married, 8,515 were widowed, 7,927 never married, 3,936 divorced and 441 were separated. Significant differences

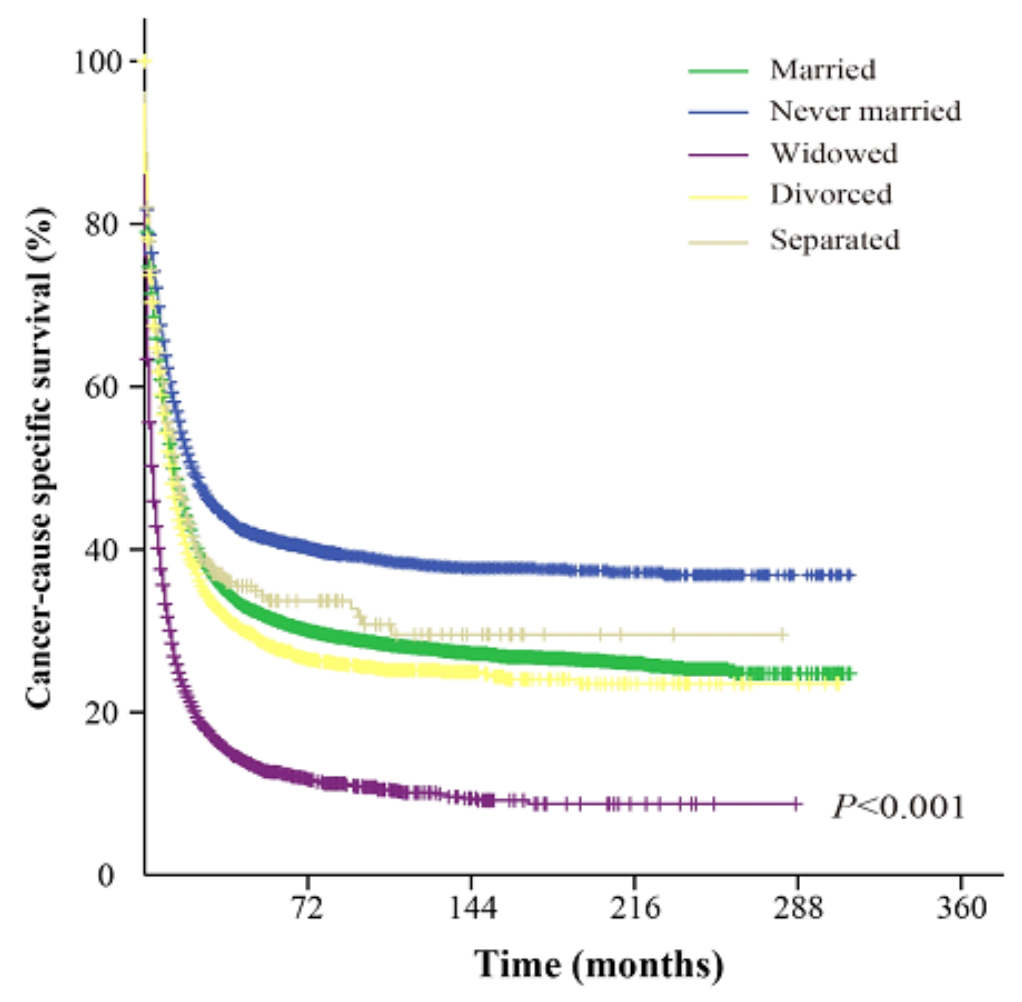

Figure 1: Survival curves of AML patients based on their marital status. $\chi^{2}=2097.9$. 
Table 1: Characteristics of AML patients based on marital status $(\boldsymbol{n}=\mathbf{5 0 8 2 5})^{\text {a }}$

\begin{tabular}{|c|c|c|c|c|c|c|c|}
\hline \multirow[t]{2}{*}{ Characteristic } & \multirow{2}{*}{$\begin{array}{c}\text { All patients no. } \\
(\%)\end{array}$} & \multicolumn{6}{|c|}{ Marital status } \\
\hline & & $\begin{array}{c}\text { Married no. } \\
(\%) \\
\end{array}$ & $\begin{array}{c}\text { Widowed no. } \\
(\%) \\
\end{array}$ & $\begin{array}{c}\text { Never married no. } \\
(\%) \\
\end{array}$ & $\begin{array}{c}\text { Divorced no. } \\
(\%) \\
\end{array}$ & $\begin{array}{c}\text { Separated no. } \\
(\%) \\
\end{array}$ & $P$-value ${ }^{\text {b }}$ \\
\hline No. of patients & $50825(100)$ & $30006(100)$ & $8515(100)$ & $7927(100)$ & $3936(100)$ & $441(100)$ & - \\
\hline Sex & & & & & & & $<0.001$ \\
\hline Male & $27510(54.1)$ & $18990(63.3)$ & $2238(26.3)$ & $4248(53.6)$ & $1809(46.0)$ & $215(48.8)$ & \\
\hline Female & $23315(45.9)$ & $11016(36.7)$ & $6267(73.7)$ & $3679(46.4)$ & $2127(54.0)$ & $226(51.2)$ & \\
\hline Age & & & & & & & $<0.001$ \\
\hline $16-35$ & $4540(8.9)$ & $1529(5.1)$ & $10(0.1)$ & $2812(35.5)$ & $137(3.5)$ & $52(11.8)$ & \\
\hline $36-55$ & $9399(18.5)$ & $6182(20.6)$ & $153(1.8)$ & $1916(24.2)$ & $1010(25.7)$ & $138(31.3)$ & \\
\hline $56-75$ & $20516(40.4)$ & $13915(46.4)$ & $2303(27.0)$ & $2131(26.9)$ & $1995(50.7)$ & $172(39.0)$ & \\
\hline $76-95$ & $16176(31.8)$ & $8354(27.8)$ & $5903(69.3)$ & $1047(13.2)$ & $793(20.1)$ & $79(17.9)$ & \\
\hline$>95$ & $194(0.4)$ & $26(0.1)$ & $146(1.8)$ & $21(0.2)$ & $1(0.0)$ & $0(0.0)$ & \\
\hline Race & & & & & & & $<0.001$ \\
\hline Black & $4102(8.1)$ & $1646(5.5)$ & $655(7.7)$ & $1261(15.9)$ & $466(11.8)$ & $74(16.8)$ & \\
\hline White & $42610(83.8)$ & $25746(85.8)$ & $7323(86.0)$ & $5927(74.8)$ & $3281(83.4)$ & $333(75.5)$ & \\
\hline Other (American Indian/AK Native, Asian/Pacific Islander) & $4013(7.9)$ & $2567(8.6)$ & $528(6.2)$ & $703(8.9)$ & 182(4.6) & $33(7.5)$ & \\
\hline Unknown & $100(0.2)$ & $47(0.1)$ & $9(0.1)$ & $36(0.4)$ & $7(0.2)$ & $1(0.2)$ & \\
\hline AML subtype & & & & & & & $<0.001$ \\
\hline AML, NOS & $30005(59.0)$ & $17442(58.1)$ & $5702(67.0)$ & $4317(54.5)$ & $2301(58.5)$ & $243(55.1)$ & \\
\hline Acute promyelocytic leukemia & $3833(7.5)$ & $2284(7.6)$ & $287(3.4)$ & $951(12.0)$ & $271(6.9)$ & $40(9.1)$ & \\
\hline Acute myelomonocytic leukemia & $4942(9.7)$ & $2958(9.9)$ & $801(9.4)$ & $757(9.5)$ & $378(9.6)$ & $48(10.9)$ & \\
\hline AML with RPN1/EVI1 fusion gene & $22(0.0)$ & $13(0.0)$ & $4(0.0)$ & $3(0.0)$ & $0(0)$ & $2(0.6)$ & \\
\hline AML with CBFB/MYH11 fusion gene & $503(1.0)$ & $283(0.9)$ & $30(0.4)$ & $126(1.6)$ & $56(1.4)$ & $8(1.8)$ & \\
\hline $\begin{array}{l}\text { AML with RUNX1/RUNX1T1 fusion gene } \\
\text { AML with MLLT3/MLL fusion gene }\end{array}$ & $\begin{array}{l}619(1.2) \\
280(0.6)\end{array}$ & $\begin{array}{l}353(1.2) \\
141(0.5)\end{array}$ & $\begin{array}{l}54(0.6) \\
44(0.5)\end{array}$ & $\begin{array}{l}142(1.8) \\
65(0.8)\end{array}$ & $\begin{array}{l}61(1.5) \\
27(0.7)\end{array}$ & $\begin{array}{l}9(1.8) \\
3(0.7)\end{array}$ & \\
\hline AML with RBM15/MKL1 fusion gene & $21(0.0)$ & $14(0.0)$ & $2(0.0)$ & $3(0.0)$ & $2(0.0)$ & $0(0.0)$ & \\
\hline AML with DEK/NUP214 fusion gene & $28(0.0)$ & $15(0.0)$ & $3(0.0)$ & $10(0.0)$ & $0(0.0)$ & $0(0.0)$ & \\
\hline AML with minimal differentiation & $1203(2.4)$ & $703(2.3)$ & $224(2.6)$ & $182(2.3)$ & $87(2.2)$ & $7(1.6)$ & \\
\hline AML without maturation & $1922(3.8)$ & $1161(3.9)$ & $274(3.2)$ & $329(4.2)$ & $146(3.7)$ & $12(2.7)$ & \\
\hline AML with maturation & $2243(4.4)$ & $1325(4.4)$ & $322(3.8)$ & $373(4.7)$ & 196(5.0) & $27(6.1)$ & \\
\hline AML with myelodysplasia-related changes & $3132(6.2)$ & $2009(6.9)$ & $515(6.0)$ & $356(4.5)$ & $228(5.8)$ & $24(6.1)$ & \\
\hline Acute erythroid leukemia & $864(1.7)$ & $546(1.8)$ & $122(1.4)$ & $112(1.4)$ & $78(2.0)$ & $6(1.4)$ & \\
\hline Acute megakaryoblastic leukemia & $312(0.6)$ & $190(0.6)$ & $38(0.4)$ & $59(0.7)$ & $22(0.6)$ & $3(0.7)$ & \\
\hline Therapy-related myeloid neoplasm & $896(1.9)$ & $569(1.9)$ & $95(1.3)$ & $142(2.0)$ & $79(2.1)$ & $11(2.4)$ & \\
\hline Adjuvant therapy & & & & & & & $<0.001$ \\
\hline None & $48683(95.8)$ & $2867(95.6)$ & $8390(98.5)$ & $7430(93.7)$ & $3772(95.8)$ & $420(95.2)$ & \\
\hline Beam radiation or radioisotopes & $1896(3.7)$ & $1201(4.0)$ & $64(0.8)$ & $467(5.9)$ & $146(3.7)$ & $18(4.1)$ & \\
\hline Unknown & $246(0.5)$ & $134(0.4)$ & $61(0.7)$ & $30(0.4)$ & $18(0.5)$ & $3(0.7)$ & \\
\hline Cause of Death & & & & & & & $<0.001$ \\
\hline Alive or dead of other cause & $11317(22.3)$ & $6653(22.2)$ & $895(10.5)$ & $2819(35.6)$ & $829(21.1)$ & $121(27.4)$ & \\
\hline Dead (attributable to AML) & $27406(53.9)$ & $15891(53.0)$ & $5285(62.1)$ & $3829(48.3)$ & $2170(55.1)$ & $231(52.4)$ & \\
\hline Not first tumor & $12102(23.8)$ & $7462(24.8)$ & $2335(27.4)$ & $1279(16.1)$ & $937(23.8)$ & $89(20.2)$ & \\
\hline
\end{tabular}

Abbreviation: NOS, no other specific; SEER, Surveillance, Epidemiology and End Results; AML, acute myeloid leukemia. aData represented number of patients.

${ }^{\mathrm{b}} P$ value of the Chi-square test or Wilcoxon-Mann-Whitney test refers to comparison for the differences in proportions among subgroups.

in demographic and clinicopathological characteristics, including sex, race, age at diagnosis, AML subtype, adjuvant therapy and cause of death were observed in patients from marital status groups. Especially, married and never married patients were more likely to be males compared in other groups. Also the married patients had a better chance to be in age groups of 36-55, 56-75, and $76-95$, while patients in younger age group of $16-35$, were predominantly never married. Most of the patients were whites. In addition, patients of all marital status groups were mostly diagnosed for AML, NOS and experienced no radiotherapy, and died due to AML. The demographic and clinicopathological characteristics of AML patients with different marital status have been summarized in Table 1.

\section{Effect of marital status and other variables on cancer-caused specific survival}

The univariate analysis showed that never married group AML patients had better cancer-CSS than married, widowed, divorced and separated patients. The median 
Table 2: Univariate and multivariate survival analysis of AML patients from SEER database

\begin{tabular}{|c|c|c|c|c|}
\hline \multirow[t]{2}{*}{ Variable } & \multicolumn{2}{|c|}{ Univariate analysis } & \multicolumn{2}{|c|}{ Multivariate analysis } \\
\hline & HR $(95 \%$ CI $)$ & $\boldsymbol{P}$ & HR $(95 \% \mathrm{CI})$ & $P$ \\
\hline Sex (Male $v s$. Female) & $1.068(1.043-1.094)$ & $<0.001$ & $1.085(1.058-1.111)$ & $<0.001$ \\
\hline Age (> 55 vs. $16-55$ years) & $2.428(2.359-2.500)$ & $<0.001$ & $2.352(2.283-2.423)$ & $<0.001$ \\
\hline Race (Non-White $v s$. White) & $0.912(0.883-0.942)$ & $<0.001$ & $0.987(0.955-1.020)$ & 0.426 \\
\hline AML subtype (Others vs. AML, NOS) & $0.674(0.658-0.691)$ & $<0.001$ & $0.727(0.709-0.745)$ & $<0.001$ \\
\hline Adjuvant therapy (Radiation vs. None ) & $0.578(0.543-0.616)$ & $<0.001$ & $0.751(0.704-0.800)$ & $<0.001$ \\
\hline Marital Status (Married $v s$. Unmarried) & $0.875(0.855-0.897)$ & $<0.001$ & $0.802(0.782-0.822)$ & $<0.001$ \\
\hline \multicolumn{5}{|l|}{ Age (years) } \\
\hline $16-35$ & Reference & & Reference & \\
\hline $36-55$ & $1.426(1.348-1.507)$ & $<0.001$ & $1.426(1.342-1.508)$ & $<0.001$ \\
\hline $56-75$ & $1.592(1.552-1.634)$ & $<0.001$ & $2.331(2.205-2.464)$ & $<0.001$ \\
\hline $76-95$ & $1.648(1.618-1.679)$ & $<0.001$ & $3.899(3.677-4.134)$ & $<0.001$ \\
\hline$>95$ & $1.634(1.557-1.715)$ & $<0.001$ & $5.603(4.665-6.730)$ & $<0.001$ \\
\hline \multicolumn{5}{|l|}{ Race } \\
\hline Black & $0.924(0.884-0.966)$ & $<0.001$ & $1.008(0.963-1.054)$ & 0.740 \\
\hline White & Reference & & Reference & \\
\hline Other (American Indian/AK Native, Asian/Pacific Islander) & $0.952(0.932-0.974)$ & $<0.001$ & $1.024(0.980-1.070)$ & 0.297 \\
\hline Unknown & $0.855(0.770-0.950)$ & 0.003 & $0.831(0.607-1.138)$ & 0.249 \\
\hline \multicolumn{5}{|l|}{ AML subtype } \\
\hline AML, NOS & Reference & & Reference & \\
\hline Acute promyelocytic leukemia & $0.295(0.277-0.314)$ & $<0.001$ & $0.375(0.352-0.399)$ & $<0.001$ \\
\hline Acute myelomonocytic leukemia & $0.984(0.965-1.003)$ & 0.094 & $1.056(1.015-1.097)$ & 0.006 \\
\hline AML with RPN1/EVIl fusion gene & $0.887(0.713-1.102)$ & 0.279 & $0.795(0.413-1.527)$ & 0.490 \\
\hline $\begin{array}{l}\text { AML with CBFB/MYH11 fusion gene } \\
\text { AML with RUNX1/RUNX1T1 fusion gene }\end{array}$ & $\begin{array}{l}0.773(0.744-0.804) \\
0.867(0.846-0.889)\end{array}$ & $\begin{array}{l}<0.001 \\
<0.001\end{array}$ & $\begin{array}{l}0.465(0.398-0.542) \\
0.607(0.536-0.687)\end{array}$ & $\begin{array}{l}<0.001 \\
<0.001\end{array}$ \\
\hline AML with MLLT3/MLL fusion gene & $0.942(0.915-0.970)$ & $<0.001$ & $0.820(0.690-0.975)$ & 0.025 \\
\hline AML with RBM15/MKL1 fusion gene & $0.910(0.819-1.012)$ & 0.082 & $0.581(0.277-1.220)$ & 0.151 \\
\hline AML with DEK/NUP214 fusion gene & $0.982(0.946-1.020)$ & 0.349 & $0.863(0.490-1.519)$ & 0.609 \\
\hline AML with minimal differentiation & $1.006(0.997-1.015)$ & 0.188 & $1.070(0.995-1.152)$ & 0.070 \\
\hline AML without maturation & $0.978(0.971-0.984)$ & $<0.001$ & $0.913(0.859-0.970)$ & 0.003 \\
\hline AML with maturation & $0.977(0.972-0.983)$ & $<0.001$ & $0.854(0.807-0.903)$ & $<0.001$ \\
\hline AML with myelodysplasia-related changes & $0.978(0.973-0.983)$ & $<0.001$ & $0.716(0.679-0.756)$ & $<0.001$ \\
\hline Acute erythroid leukemia & $0.993(0.986-1.001)$ & 0.087 & $0.901(0.822-0.987)$ & 0.026 \\
\hline Acute megakaryoblastic leukemia & $1.009(0.998-1.020)$ & 0.095 & $1.216(1.055-1.402)$ & 0.007 \\
\hline Therapy-related myeloid neoplasm & $0.810(0.789-0.830)$ & $<0.001$ & $0.055(0.038-0.078)$ & $<0.001$ \\
\hline \multicolumn{5}{|l|}{ Adjuvant therapy } \\
\hline None & Reference & & Reference & \\
\hline Beam radiation or radioisotopes & $0.524(0.490-0.562)$ & $<0.001$ & $0.703(0.655-0.754)$ & $<0.001$ \\
\hline Unknown & $1.136(1.050-1.229)$ & 0.002 & $1.114(0.952-1.304)$ & 0.179 \\
\hline \multicolumn{5}{|l|}{ Marital Status } \\
\hline Married & Reference & & Reference & \\
\hline Widowed & $1.813(1.757-1.871)$ & $<0.001$ & $1.312(1.267-1.357)$ & $<0.001$ \\
\hline Never married & $0.786(0.758-0.814)$ & $<0.001$ & $1.167(1.125-1.211)$ & $<0.001$ \\
\hline Divorced & $1.072(1.025-1.122)$ & 0.002 & $1.148(1.098-1.201)$ & $<0.001$ \\
\hline Separated & $0.942(0.827-1.073)$ & 0.268 & $1.145(1.006-1.304)$ & 0.041 \\
\hline
\end{tabular}

Abbreviation: CSS, cancer-specific death; HR, hazard ratio; CI, confidence interval; AML, acute myeloid leukemia; NOS, no other specific; SEER, Surveillance, Epidemiology and End Results

cancer-CSS in this group was 22 months, while it was 13 months in married group, 14 months in separated group, 4 months in widowed group and 12 months in divorced group patients. This difference was statistically significant according to the univariate log-rank test $(P<$ 0.001) (Figure. 1). In addition, among the demographic and clinicopathological variables, sex, age, race, AML subtype, adjuvant therapy, and marital status were identified as independent factors for predicting CSS based on univariate analysis (Table 2). However, multivariate analysis with Cox regression model indicated sex (male, HR 1.085, 95\%CI: 1.058-1.111, $P<0.001$ ), age ( $>55$ years, HR 2.352, 95\%CI: 2.283-2.423, $P<0.001$ ), AML subtype (others, HR 0.727, 95\%CI: 0.709-0.745, $P<$ 0.001 ), adjuvant therapy (radiation, HR $0.751,95 \% \mathrm{CI}$ : 0.704-0.800, $P<0.001$ ), and marital status (married, HR 
0.802, 95\%CI: $0.782-0.822, P<0.001)$ as independent prognostic factors. In addition, further multivariate analysis based on subgroups like age, AML subtype and marital status, again validated these independent prognostic factors. For example, age, 36-55 years (HR $1.426,95 \% \mathrm{CI}: 1.342-1.508, P<0.001)$; age, $56-75$ years (HR 2.331, 95\%CI: 2.205-2.464, $P<0.001$ ); age, 7695 years (HR 3.899, 95\%CI: 3.677-4.134, $P<0.001$ ); age, $>95$ years (HR 5.603, 95\%CI: 4.665-6.730, $P<$ $0.001)$; diagnosed as acute promyelocytic leukemia (HR 0.375, 95\%CI: 0.352-0.399, $P<0.001$ ); diagnosed as acute myelomonocytic leukemia (HR 1.056, 95\%CI: 1.015-1.097, $P=0.006$ ); diagnosed as AML with $\mathrm{CBFB} /$ MYH11 fusion gene (HR 0.465, 95\%CI: 0.398-0.542, $P$ $<0.001)$; diagnosed as AML with RUNX1/RUNX1T1 fusion gene (HR 0.607, 95\%CI: 0.536-0.687, $P<0.001$ ); diagnosed as AML with MLLT3/MLL fusion gene (HR 0.820, 95\%CI: $0.690-0.975, P=0.025)$; diagnosed as AML without maturation (HR 0.913 , 95\%CI: 0.859 $0.970, P=0.003)$; diagnosed as AML with maturation (HR 0.854, 95\%CI: 0.807-0.903, $P<0.001$ ); diagnosed as AML with myelodysplasia-related changes (HR 0.716, 95\%CI: $0.679-0.756, P<0.001)$; diagnosed as acute erythroid leukemia (HR 0.901, 95\%CI: 0.822-0.987, $P$ $=0.026$ ); diagnosed as acute megakaryoblastic leukemia (HR 1.216, 95\%CI: 1.055-1.402, $P=0.007$ ); diagnosed as therapy-related myeloid neoplasm (HR 0.055 95\%CI: 0.038-0.078, $P=0.007$ ); and marital status (widowed, HR 1.312, 95\%CI: $1.267-1.357, P<0.001$; never married, HR 1.167, 95\% CI: 1.125-1.21, $P<0.001$; divorced, HR 1.148, 95\%CI: $1.098-1.201, P<0.001$ and separated, HR 1.145, 95\%CI: $1.006-1.304, P=0.041)$.

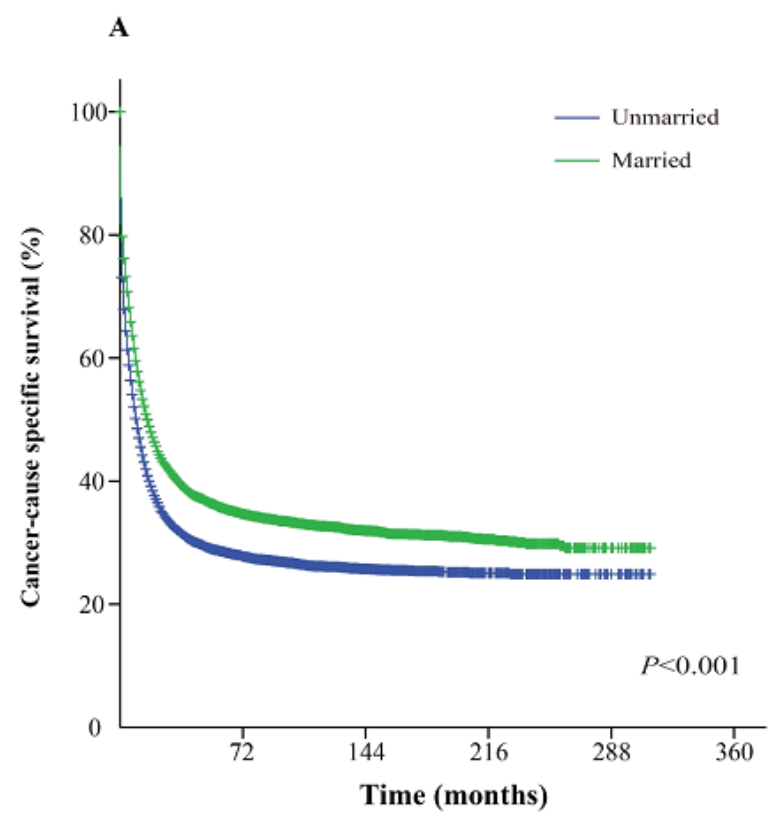

\section{Subgroup analysis of the marital status effect on cancer-CSS based on sex, age, AML subtype and adjuvant therapy}

Our study has identified multiple variables including, sex, age, AML subtype and adjuvant therapy as risk factors for AML mortality, based on Cox proportional hazard regression model analysis. This observation has also been verified independently by other studies [19-21]. Thus, subsequently we stratified patients from different marital status into subgroups based on these variables and studied their effect on cancer-CSS. Our analysis revealed that patients from widowed and never married groups correlated with poor CSS, in comparison to married group, after all these patients were adjusted for the aforementioned variables $(P<0.05)$. In addition, divorced group patients also displayed increased risk for cancer-caused specific mortality, in comparison to married group patients, after adjustment for all variables except in radiation subgroup (HR 1.134, 95\% CI: 0.898-1.430, $P$ $=0.291$ ). However, the difference between married and separated group patients was not apparent in the majority of the subgroups (Table 3).

\section{Analysis of cancer-caused specific survival between matched groups}

Based on the demographic and clinicopathological variables, propensity scores for unmarried, never married, widowed and divorced patient groups were estimated.

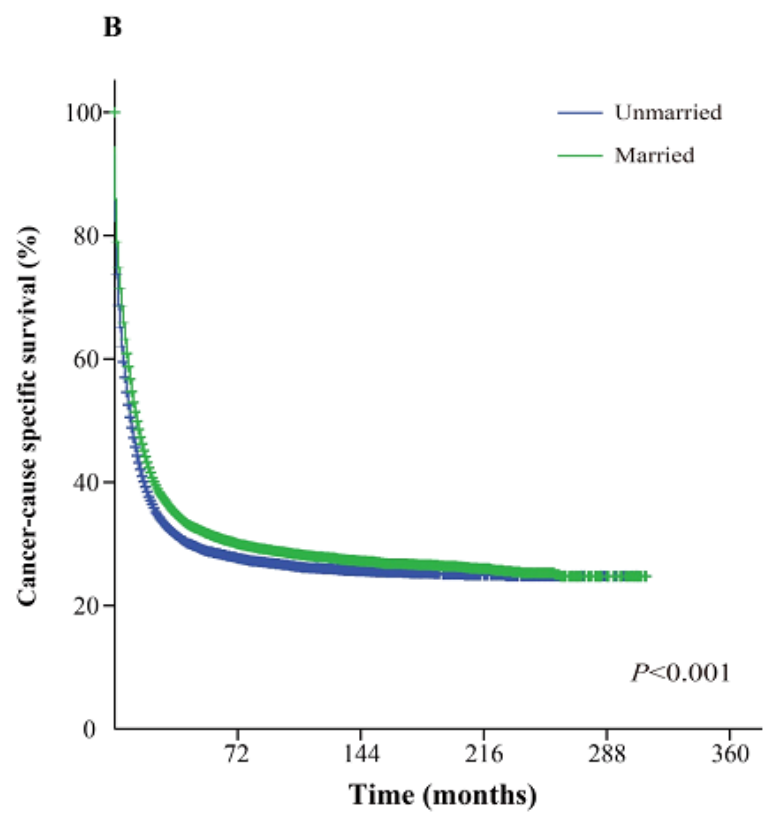

Figure 2: Kaplan-Meier survival curves: The cancer-caused specific survival of unmarried and married groups of matched and unmatched AML patients. A. matched group, $\chi^{2}=295.5 ;$ B. unmatched group, $\chi^{2}=128.1$. 
Table 3: Univariate and multivariate CSS analysis, based on sex, age, AML subtype and adjuvant therapy, of AML patients with different marital status

\begin{tabular}{|c|c|c|c|c|}
\hline \multirow[t]{2}{*}{ Variable } & \multicolumn{2}{|c|}{ Univariate analysis } & \multicolumn{2}{|c|}{ Multivariate analysis } \\
\hline & HR $(95 \%$ CI) & $P$ & HR $(95 \% \mathrm{CI})$ & $P$ \\
\hline \multicolumn{5}{|l|}{ Sex } \\
\hline \multicolumn{5}{|l|}{ Male } \\
\hline Married & Reference & & Reference & \\
\hline Widowed & $1.623(1.533-1.719)$ & $<0.001$ & $1.197(1.129-1.296)$ & $<0.001$ \\
\hline Never married & $0.763(0.729-0.800)$ & $<0.001$ & $1.157(1.101-1.215)$ & $<0.001$ \\
\hline Divorced & $1.098(1.031-1.170)$ & 0.004 & $1.179(1.106-1.256)$ & $<0.001$ \\
\hline Separated & $0.976(0.814-1.170)$ & 0.790 & $1.164(0.971-1.396)$ & 0.101 \\
\hline \multicolumn{5}{|l|}{ Female } \\
\hline Married & Reference & & Reference & \\
\hline Widowed & $2.115(2.029-2.205)$ & $<0.001$ & $1.318(1.260-1.379)$ & $<0.001$ \\
\hline Never married & $0.846(0.801-0.894)$ & $<0.001$ & $1.165(1.102-1.232)$ & $<0.001$ \\
\hline Divorced & $1.131(1.061-1.207)$ & $<0.001$ & $1.121(1.051-1.195)$ & 0.001 \\
\hline Separated & $0.976(0.810-1.176)$ & 0.797 & $1.131(0.939-1.363)$ & 0.196 \\
\hline \multicolumn{5}{|l|}{ Age (years) } \\
\hline \multicolumn{5}{|l|}{$16-55$} \\
\hline Married & Reference & & Reference & \\
\hline Widowed & $1.320(1.066-1.635)$ & 0.011 & $1.320(1.065-1.636)$ & 0.011 \\
\hline Never married & $0.981(0.928-1.037)$ & 0.501 & $1.146(1.079-1.218)$ & $<0.001$ \\
\hline Divorced & $1.273(1.166-1.390)$ & $<0.001$ & $1.265(1.158-1.382)$ & $<0.001$ \\
\hline Separated & $1.116(0.903-1.380)$ & 0.308 & $1.176(0.952-1.453)$ & 0.134 \\
\hline \multicolumn{5}{|l|}{$>55$} \\
\hline Married & Reference & & Reference & \\
\hline Widowed & $1.505(1.457-1.555)$ & $<0.001$ & $1.279(1.233-1.326)$ & $<0.001$ \\
\hline Never married & $1.111(1.058-1.167)$ & $<0.001$ & $1.139(1.085-1.197)$ & $<0.001$ \\
\hline Divorced & $1.043(0.990-1.099)$ & 0.115 & $1.116(1.059-1.176)$ & $<0.001$ \\
\hline Separated & $1.071(0.098-1.262)$ & 0.417 & $1.123(0.952-1.324)$ & 0.169 \\
\hline \multicolumn{5}{|l|}{ AML subtype } \\
\hline \multicolumn{5}{|l|}{ AML, NOS } \\
\hline Married & Reference & & Reference & \\
\hline Widowed & $1.745(1.679-1.813)$ & $<0.001$ & $1.321(1.267-1.377)$ & $<0.001$ \\
\hline Never married & $0.811(0.775-0.849)$ & $<0.001$ & $1.157(1.104-1.213)$ & $<0.001$ \\
\hline Divorced & $1.078(1.019-1.141)$ & 0.009 & $1.154(1.090-1.222)$ & $<0.001$ \\
\hline Separated & $1.038(0.881-1.223)$ & 0.654 & $1.255(1.065-1.478)$ & 0.007 \\
\hline \multicolumn{5}{|l|}{ Others } \\
\hline Married & Reference & & Reference & \\
\hline Widowed & $1.827(1.728-1.932)$ & $<0.001$ & $1.292(1.216-1.372)$ & $<0.001$ \\
\hline Never married & $0.771(0.728-0.816)$ & $<0.001$ & $1.180(1.112-1.253)$ & $<0.001$ \\
\hline Divorced & $1.064(0.989-1.146)$ & 0.098 & $1.135(1.054-1.222)$ & 0.001 \\
\hline Separated & $0.852(0.689-1.054)$ & 0.141 & $1.040(0.840-1.287)$ & 0.719 \\
\hline \multicolumn{5}{|l|}{ Adjuvant therapy } \\
\hline \multicolumn{5}{|l|}{ None } \\
\hline Married & Reference & & Reference & \\
\hline Widowed & $1.763(1.708-1.820)$ & $<0.001$ & $1.299(1.255-1.345)$ & $<0.001$ \\
\hline Never married & $0.788(0.760-0.817)$ & $<0.001$ & $1.157(1.114-1.201)$ & $<0.001$ \\
\hline Divorced & $1.066(1.019-1.116)$ & 0.006 & $1.148(1.097-1.203)$ & $<0.001$ \\
\hline Separated & $0.929(0.813-1.061)$ & 0.276 & $1.119(0.979-1.278)$ & 0.100 \\
\hline \multicolumn{5}{|c|}{ Beam radiation or radioisotopes } \\
\hline Married & Reference & & Reference & \\
\hline Widowed & $3.049(2.430-3.827)$ & $<0.001$ & $1.483(1.148-1.916)$ & 0.003 \\
\hline Never married & $0.874(0.748-1.020)$ & 0.088 & $1.252(1.052-1.489)$ & 0.011 \\
\hline Divorced & $1.170(0.928-1.475)$ & 0.185 & $1.134(0.898-1.430)$ & 0.291 \\
\hline Separated & $1.289(0.728-2.283)$ & 0.384 & $1.865(1.049-3.318)$ & 0.034 \\
\hline
\end{tabular}

Abbreviation: CSS, cancer-specific death; HR, hazard ratio; CI, confidence interval; AML, acute myeloid leukemia; NOS, no other specific; 
Table 4: Baseline characteristics of unmarried and married AML patients, before and after propensity matching

\begin{tabular}{|c|c|c|c|c|c|c|c|c|}
\hline \multirow[t]{2}{*}{ Variable } & \multicolumn{2}{|c|}{ Unmatched (complete) dataset } & \multirow[t]{2}{*}{$\chi^{2}$} & \multirow[t]{2}{*}{$P$} & \multicolumn{2}{|c|}{ Matched (1:1) dataset } & \multirow[t]{2}{*}{$\chi^{2}$} & \multirow[t]{2}{*}{$P$} \\
\hline & $\begin{array}{l}\text { Unmarried } \\
(\mathrm{n}=\mathbf{2 0 8 1 9})\end{array}$ & married $(n=30006)$ & & & Unmarried $(n=18346)$ & married $(n=18345)$ & & \\
\hline Age (years) & & & 109.803 & $<0.001$ & & & 0.101 & 0.751 \\
\hline $16-55$ & $6228(29.9 \%)$ & $7711(25.7 \%)$ & & & $5787(31.5 \%)$ & $5815(31.7 \%)$ & & \\
\hline$>55$ & $14591(70.1 \%)$ & $22295(74.3 \%)$ & & & $12559(68.5 \%)$ & $12530(86.3 \%)$ & & \\
\hline Race & & & 686.708 & $<0.001$ & & & 0.729 & 0.866 \\
\hline Black & $2456(11.8 \%)$ & $1646(5.5 \%)$ & & & $1464(8.0 \%)$ & $1431(7.8 \%)$ & & \\
\hline White & $16864(81.0 \%)$ & $25746(85.8 \%)$ & & & $15440(84.2 \%)$ & $15442(84.2 \%)$ & & \\
\hline Other & $1446(6.9 \%)$ & $2567(8.6 \%)$ & & & $1411(7.7 \%)$ & $1442(7.9 \%)$ & & \\
\hline Unknown & $53(0.3 \%)$ & $47(0.1 \%)$ & & & $31(0.1 \%)$ & $30(0.1 \%)$ & & \\
\hline Sex & & & 2475.636 & $<0.001$ & & & 0.000 & 1.000 \\
\hline Male & $8520(40.9 \%)$ & $18990(63.3 \%)$ & & & $8380(45.7 \%)$ & $8379(45.7 \%)$ & & \\
\hline Female & $12299(59.1 \%)$ & $11016(36.7 \%)$ & & & $9966(54.3 \%)$ & $9966(54.3 \%)$ & & \\
\hline AML subtype & & & 24.948 & $<0.001$ & & & 0.097 & 0.755 \\
\hline AML, NOS & $12563(60.3 \%)$ & $17442(58.1 \%)$ & & & $10705(58.4 \%)$ & $10675(58.2 \%)$ & & \\
\hline other & $8256(39.7 \%)$ & $12564(41.9 \%)$ & & & $7641(41.6 \%)$ & $7670(41.8 \%)$ & & \\
\hline Adjuvant therapy & & & $17 . .079$ & $<0.001$ & & & 0.743 & 0.690 \\
\hline None & $20012(96.1 \%)$ & $28671(95.6 \%)$ & & & $17603(96.0 \%)$ & $17572(95.8 \%)$ & & \\
\hline Beam radiation or radioisotopes & $695(3.3 \%)$ & $1201(4.0 \%)$ & & & $662(3.6 \%)$ & $693(3.8 \%)$ & & \\
\hline Unknown & $112(0.6 \%)$ & $134(0.4 \%)$ & & & $81(0.4 \%)$ & $80(0.4 \%)$ & & \\
\hline
\end{tabular}

Abbreviation: AML, acute myeloid leukemia; NOS, no other specific.

Next, the patients were independently matched based on their propensity scores with the married group patients. For instance, the 1:1 matching between unmarried and married group patients resulted in 18,345 matched pairs and a sample size of 36,691 patients. Similarly, the matching between never married and married patients resulted in, 7,697 matched pairs. All the group differences in both these matched datasets did not reach the statistically significance $(P>0.05)$, thereby, representing negligible differences across AML subtypes and all other demographic and treatment variables (Table 4, 5). However, in case of matching of patients from widowed group with married patients, race and adjuvant therapy were categorized as a binary variables to minimize the
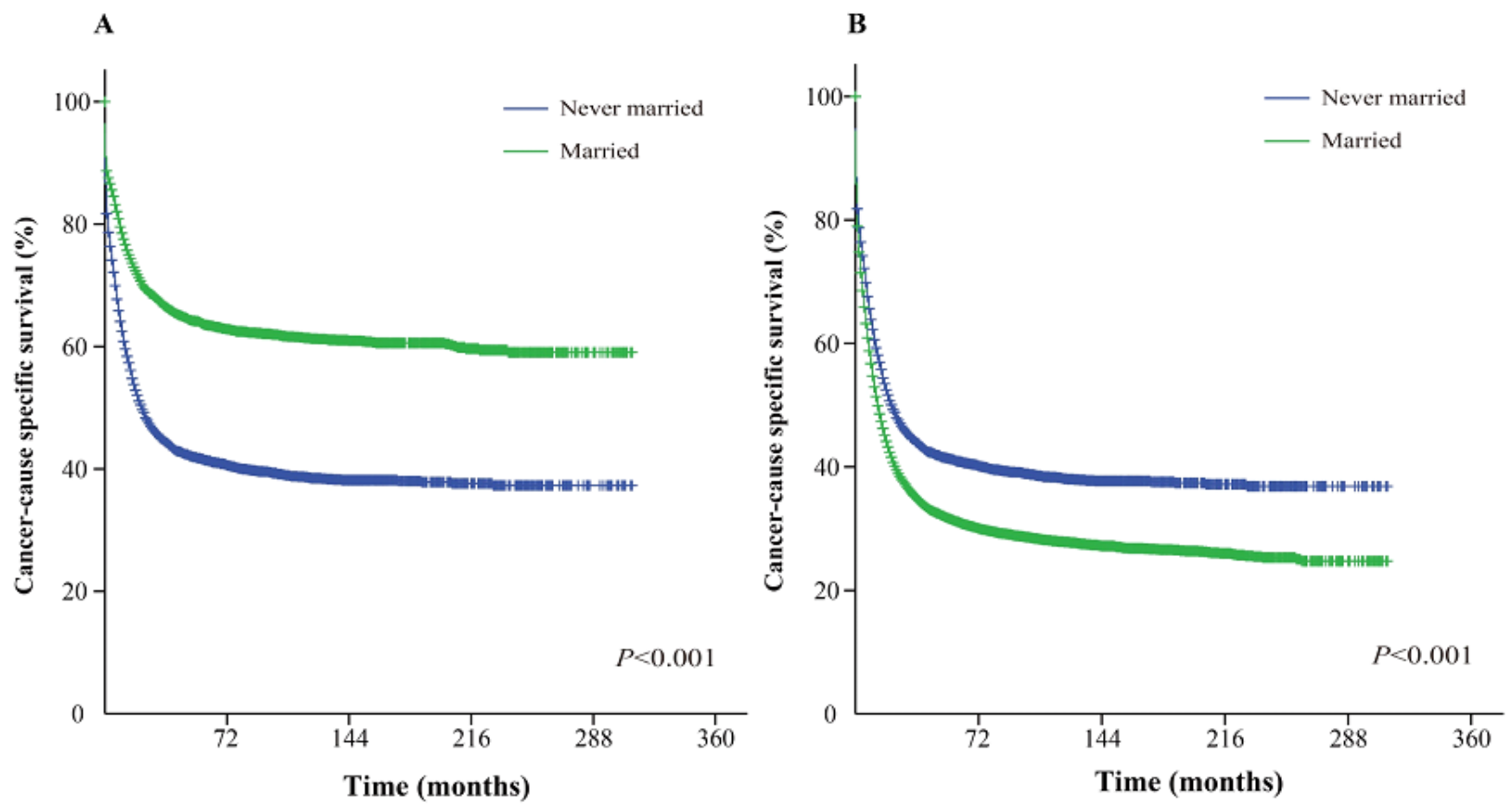

Figure 3: Kaplan-Meier survival curves: The cancer-caused specific survival of never married and married groups of matched and unmatched AML patients. A. matched group, $\chi^{2}=639.6 ;$ B. unmatched group, $\chi^{2}=191.4$. 
Table 5: Baseline characteristics of never married and married AML patients, before and after propensity matching

\begin{tabular}{|c|c|c|c|c|c|c|c|c|}
\hline \multirow[t]{2}{*}{ Variable } & \multicolumn{2}{|c|}{ Unmatched (complete) dataset } & \multirow[t]{2}{*}{$\chi^{2}$} & \multirow[t]{2}{*}{$P$} & \multicolumn{2}{|c|}{ Matched (1:1) dataset } & \multirow[t]{2}{*}{$\chi^{2}$} & \multirow[t]{2}{*}{$P$} \\
\hline & never married $(n=7927)$ & $\operatorname{married}(n=30006)$ & & & never married $(n=7697)$ & $\operatorname{married}(n=7697)$ & & \\
\hline Age (years) & & & 3278.608 & $<0.001$ & & & 0.000 & 1.000 \\
\hline $16-55$ & $4728(59.6 \%)$ & $7711(25.7 \%)$ & & & $4502(58.5 \%)$ & $4502(58.5 \%)$ & & \\
\hline$>55$ & $3199(40.4 \%)$ & $22295(74.3 \%)$ & & & $3195(41.5 \%)$ & $3195(41.5 \%)$ & & \\
\hline Race & & & 1006.249 & $<0.001$ & & & 0.346 & 0.951 \\
\hline Black & $1261(15.9 \%)$ & $1646(5.5 \%)$ & & & $1043(13.6 \%)$ & $1043(13.6 \%)$ & & \\
\hline White & $5927(74.8 \%)$ & $25746(85.8 \%)$ & & & $5926(77.0 \%)$ & $5905(76.7 \%)$ & & \\
\hline Other & $703(8.9 \%)$ & $2567(8.6 \%)$ & & & $703(9.1 \%)$ & $724(9.4 \%)$ & & \\
\hline Unknown & $36(0.4 \%)$ & $47(0.1 \%)$ & & & $25(0.3 \%)$ & $25(0.3 \%)$ & & \\
\hline Sex & & & 248.520 & $<0.001$ & & & 0.116 & 0.734 \\
\hline Male & $4248(53.6 \%)$ & $18990(63.3 \%)$ & & & $3509(45.6 \%)$ & $3488(45.3 \%)$ & & \\
\hline Female & $3679(46.4 \%)$ & $11016(36.7 \%)$ & & & $4188(54.4 \%)$ & $4209(54.7 \%)$ & & \\
\hline AML subtype & & & 34.511 & $<0.001$ & & & 0.000 & 1.000 \\
\hline AML, NOS & $4317(54.5 \%)$ & $17442(58.1 \%)$ & & & $4192(54.5 \%)$ & $4192(54.5 \%)$ & & \\
\hline other & $3610(45.5 \%)$ & $12564(41.9 \%)$ & & & $3505(45.5 \%)$ & $3505(45.5 \%)$ & & \\
\hline Adjuvant therapy & & & $53 . .727$ & $<0.001$ & & & 0.588 & 0.745 \\
\hline None & $7430(93.7 \%)$ & $28671(95.6 \%)$ & & & $7216(93.8 \%)$ & $7200(93.5 \%)$ & & \\
\hline Beam radiation or radioisotopes & $467(5.9 \%)$ & $1201(4.0 \%)$ & & & $455(5.9 \%)$ & $466(6.1 \%)$ & & \\
\hline Unknown & $30(0.4 \%)$ & $134(0.4 \%)$ & & & $26(0.3 \%)$ & $31(0.4 \%)$ & & \\
\hline
\end{tabular}

Abbreviation: AML, acute myeloid leukemia; NOS, no other specific.

group difference, and this led to 8,495 matched pairs without group differences except race variable. Finally, the survival analysis was also performed for this matched dataset (Table 6). Importantly, the matching of divorced group with married group could not be done due to the absence of matched dataset across most variables (Appendix Tables 1), and thus no further survival analyses were performed.

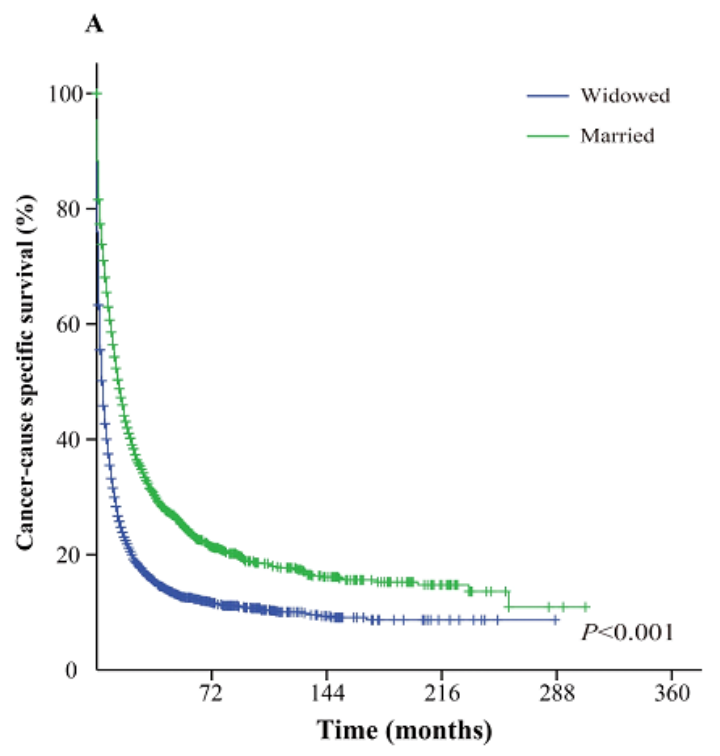

The Kaplan-Meier curve based estimation of cancercaused specific survival for the unmatched and matched marital status groups, was performed as shown in Figure $2,3 \& 4$. The survival analysis between unmatched unmarried and married group patients showed a median CSS of 10 months (95\% CI: 9.6-10.4) in the unmarried group, while 13 months (95\% CI: 12.5-13.5) in the married group patients $(P<0.001)$. A similar analysis between

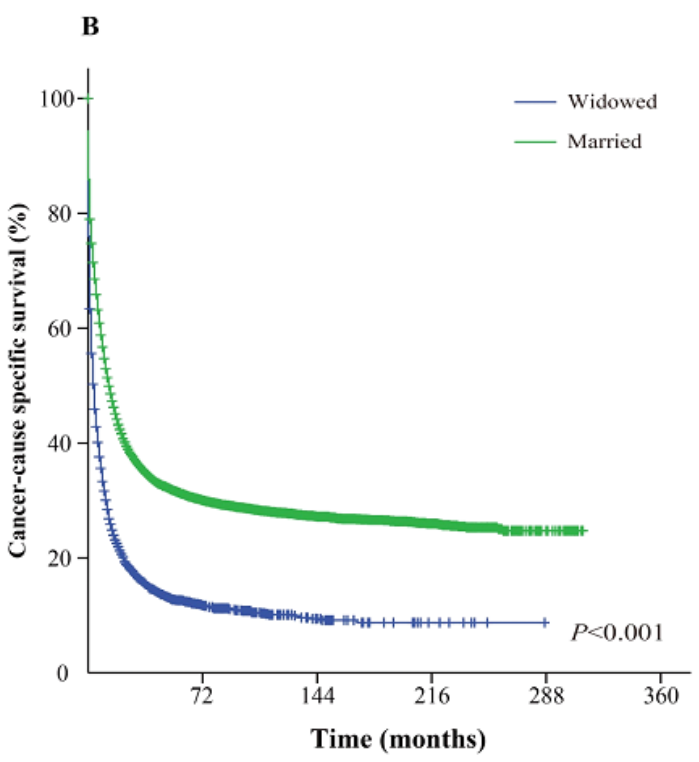

Figure 4: Kaplan-Meier survival curves: The cancer-caused specific survival of widowed and married groups of matched and unmatched AML patients. A. matched group, $\chi^{2}=891.4$; B. unmatched group, $\chi^{2}=1565.2$. 
Table 6: Baseline characteristics of widowed and married AML patients, before and after propensity matching

\begin{tabular}{|c|c|c|c|c|c|c|c|c|}
\hline \multirow[t]{2}{*}{ Variable } & \multicolumn{2}{|c|}{ Unmatched (complete) dataset } & \multirow[t]{2}{*}{$\chi^{2}$} & \multirow[t]{2}{*}{$P$} & \multicolumn{2}{|c|}{ Matched (1:1) dataset } & \multirow[t]{2}{*}{$\chi^{2}$} & \multirow[t]{2}{*}{$\boldsymbol{P}$} \\
\hline & widowed $(n=8515)$ & $\operatorname{married}(n=30006)$ & & & widowed $(n=8495)$ & married $(n=8495)$ & & \\
\hline Age (years) & & & 2307.140 & $<0.001$ & & & 0.000 & 1.000 \\
\hline $16-55$ & $163(1.9 \%)$ & $7711(25.7 \%)$ & & & $163(1.9 \%)$ & $163(1.9 \%)$ & & \\
\hline$>55$ & $8352(98.1 \%)$ & $22295(74.3 \%)$ & & & $8332(98.1 \%)$ & $8332(98.1 \%)$ & & \\
\hline Race & & & 0.215 & 0.643 & & & 5.562 & 0.018 \\
\hline Non white & $1192(14.0 \%)$ & $4260(14.2 \%)$ & & & $1172(13.8 \%)$ & $1068(12.6 \%)$ & & \\
\hline White & $7323(86.0 \%)$ & $25746(85.8 \%)$ & & & $7323(86.2 \%)$ & $7427(87.4 \%)$ & & \\
\hline Sex & & & 3648.390 & $<0.001$ & & & 0.000 & 1.000 \\
\hline Male & $2248(26.3 \%)$ & $18990(63.3 \%)$ & & & $2248(36.0 \%)$ & $2248(36.0 \%)$ & & \\
\hline Female & $6267(73.7 \%)$ & $11016(36.7 \%)$ & & & $6247(64.0 \%)$ & $6247(64.0 \%)$ & & \\
\hline AML subtype & & & 215.910 & $<0.001$ & & & 0.273 & 0.601 \\
\hline AML, NOS & $5702(67.0 \%)$ & $17442(58.1 \%)$ & & & $5682(66.9 \%)$ & $5714(67.3 \%)$ & & \\
\hline other & $2813(33.0 \%)$ & $12564(41.9 \%)$ & & & $2813(33.1 \%)$ & $2781(32.7 \%)$ & & \\
\hline Adjuvant therapy & & & 161.651 & $<0.001$ & & & 0.016 & 0.898 \\
\hline None radiation & $8390(98.5 \%)$ & $28671(95.6 \%)$ & & & $8370(98.5 \%)$ & $8372(98.6 \%)$ & & \\
\hline Beam radiation or radioisotopes & $125(1.5 \%)$ & $1335(4.4 \%)$ & & & $125(1.5 \%)$ & $123(1.4 \%)$ & & \\
\hline
\end{tabular}

Abbreviation: AML, acute myeloid leukemia; NOS, no other specific.

Table 7: Hazard ratios of CSS predictors in AML patients with different marital status, based on multivariate analysis (matched and unmatched complete datasets)

\begin{tabular}{|c|c|c|c|c|c|c|c|c|}
\hline \multirow[t]{2}{*}{ Variable } & \multicolumn{2}{|c|}{ Unmarried $v s$. married matched } & \multicolumn{2}{|c|}{$\begin{array}{c}\text { Unmarried vs. married } \\
\text { unmatched }\end{array}$} & \multicolumn{2}{|c|}{ Never married vs. married matched } & \multicolumn{2}{|c|}{$\begin{array}{c}\text { Never married } v s . \text { married } \\
\text { unmatched }\end{array}$} \\
\hline & HR $(95 \% \mathrm{CI})$ & $\boldsymbol{P}$ & HR $(95 \%$ CI $)$ & $\boldsymbol{P}$ & HR $(95 \%$ CI $)$ & $\boldsymbol{P}$ & HR $(95 \%$ CI $)$ & $P$ \\
\hline Age ( $>55$ vs. $16-55$ years) & $2.438(2.358-2.522)$ & $<0.001$ & $2.290(2.223-2.359)$ & $<0.001$ & $1.611(1.529-1.698)$ & $<0.001$ & $2.165(2.094-2.237)$ & $<0.001$ \\
\hline \multicolumn{9}{|l|}{ Race } \\
\hline Black & $0.946(0.898-0.998)$ & 0.041 & $1.003(0.959-1.048)$ & 0.912 & $1.549(1.449-1.656)$ & $<0.001$ & $1.030(0.976-1.087)$ & 0.285 \\
\hline White & Reference & & Reference & & Reference & & Reference & \\
\hline Other & $0.920(0.872-0.971)$ & 0.002 & $1.013(0.969-1.058)$ & 0.574 & $1.018(0.931-1.114)$ & 0.692 & $1.053(1.002-1.106)$ & 0.041 \\
\hline Unknown & $0.531(0.335-0.844)$ & 0.007 & $0.776(0.567-1.062)$ & 0.113 & $0.778(0.461-1.315)$ & 0.349 & $0.750(0.521-1.079)$ & 0.121 \\
\hline Sex (Male vs. Female) & $1.012(0.984-1.040)$ & 0.420 & $1.036(1.012-1.061)$ & 0.004 & $1.117(1.062-1.176)$ & $<0.001$ & $1.160(1.126-1.194)$ & $<0.001$ \\
\hline AML subtype (Others $v s$. AML, NOS) & $0.741(0.720-0.763)$ & $<0.001$ & $0.725(0.708-0.744)$ & $<0.001$ & $0.958(0.910-1.008)$ & 0.102 & $0.733(0.712-0.754)$ & $<0.001$ \\
\hline \multicolumn{9}{|l|}{ Adjuvant therapy } \\
\hline None & Reference & & Reference & & Reference & & Reference & \\
\hline Radiation & $0.683(0.627-0.743)$ & $<0.001$ & $0.693(0.646-0.743)$ & $<0.001$ & $0.844(0.755-0.944)$ & 0.003 & $0.716(0.664-0.771)$ & $<0.001$ \\
\hline Unknown & $1.172(0.966-1.418)$ & 0.101 & $1.215(1.038-1.422)$ & 0.016 & $1.286(0.861-1.921)$ & 0.219 & $1.162(0.948-1.425)$ & 0.149 \\
\hline
\end{tabular}

Abbreviation: CSS, cancer-specific death; HR, hazard ratio; CI, confidence interval; AML, acute myeloid leukemia; NOS, no other specific.

matched unmarried and married groups showed, a median CSS of 10 months (95\% CI: 9.5-10.5) and 16 months (95\% CI: 15.2-16.8) $(P<0.001$, Figure. 2) respectively. In addition, the 5-year cancer-caused specific survival between unmarried and married patients was $28.4 \%$ and $31.1 \%$ in unmatched group, while $28.6 \%$ versus $35.7 \%$ in matched, group, respectively $(P<0.001)$. The never married versus married analysis between unmatched groups showed median CSS of 22 months (95\% CI: 20.1$23.9)$ and 13 months (95\% CI: $12.5-13.5)$ respectively $(P<$ $0.001)$. On the contrary, in the matched group, the median CSS was 22 months (95\% CI: 20.0-24.0) for never married patients, while the married patients did not achieve the required $50 \%$ survival value $(P<0.001$, Figure. 3$)$. The 5 -year cancer-caused specific survival was $40.9 \%$ versus $31.1 \%$ in unmatched and $41.4 \%$ versus not reached in matched never married and married group patients $(P$ $<0.001)$. In addition, the cancer-CSS analysis between widowed and married patients displayed a median CSS of 4 months (95\% CI: 3.7-4.3) and 13 months (95\% CI: 12.5-13.5), respectively, and 5-year cancer-caused specific survival of $12.6 \%$ and $31.1 \%$ respectively in unmatched groups $(P<0.001)$. The similar trends were observed in the matched groups, where the median CSS was 4 months (95\% CI 3.7-4.3) versus 14 months (95\% CI: 13.2-14.8) in widowed versus married group patients $(P<0.001$, 
Table 8: Hazard ratios of CSS predictors by multivariate analysis in widowed vs. married AML patients (matched and unmatched)

\begin{tabular}{|c|c|c|c|c|}
\hline \multirow[t]{2}{*}{ Variable } & \multicolumn{2}{|c|}{ widowed $v s$. married matched } & \multicolumn{2}{|c|}{ widowed $v s$. married unmatched } \\
\hline & HR $(95 \%$ CI) & $P$ & HR $(95 \%$ CI $)$ & $P$ \\
\hline Sex (Male vs. Female) & $0.570(0.538-0.604)$ & $<0.001$ & $0.998(0.971-1.025)$ & 0.887 \\
\hline Age (> 55 vs. $16-55$ years) & $3.593(2.904-4.445)$ & $<0.001$ & $2.339(2.252-2.429)$ & $<0.001$ \\
\hline Race (Non-White $v s$. White) & $0.977(0.920-1.037)$ & 0.445 & $0.988(0.950-1.028)$ & 0.552 \\
\hline AML subtype (Others vs. AML, NOS) & $0.807(0.771-0.844)$ & $<0.001$ & $0.733(0.712-0.754)$ & $<0.001$ \\
\hline Adjuvant therapy (Radiation vs. None ) & $0.871(0.737-1.030)$ & 0.106 & $0.725(0.672-0.783)$ & $<0.001$ \\
\hline
\end{tabular}

Abbreviation: CSS, cancer-specific death; HR, hazard ratio; CI, confidence interval; AML, acute myeloid leukemia; NOS, no other specific.

Table 9: Comparison of HRs for overall survival associated with conventional treatments (based on prior literature), and with cancer-specific survival associated with marriage (in the present study) in AML patients

\begin{tabular}{|c|c|c|c|c|}
\hline Type of Treatment Study & $\begin{array}{c}\text { Chemotherapy or Hematopoietic stem cell } \\
\text { transplantation }\end{array}$ & Reference & $\begin{array}{l}\text { HR for Treatment } \\
\qquad(95 \% \mathrm{CI})\end{array}$ & $\begin{array}{l}\text { HR for Marriage } \\
\text { in Present Study }\end{array}$ \\
\hline Meta-Analysis of Prospective Studies [45] & Allogeneic HSCT & $\begin{array}{l}\text { Non-allogeneic HSCT (autologous HSCT or } \\
\text { chemotherapy }\end{array}$ & $0.76(0.61-0.95)$ & \\
\hline Meta-Analysis of Prospective Clinical Trials [46] & High doses of daunorubicin & Standard doses of daunorubicin or idarubicin & $0.88(0.79-0.99)$ & \\
\hline Systematic Review and Meta-analysis [47] & High dose of cytarabine & Allogeneic HSCT or autologous HSCT & $1.66(1.30-2.14)$ & \\
\hline $\begin{array}{l}\text { Meta-analysis of Prospective Randomized Phase } \\
\text { III Trials [48] }\end{array}$ & Gemtuzumab ozogamicin included regimens & $\begin{array}{l}\text { Non Gemtuzumab ozogamicin included } \\
\text { regimens }\end{array}$ & $0.93(0.86-1.00)$ & \\
\hline Systematic Review and Meta-analysis [49] & $\begin{array}{l}\text { Conventional chemotherapy with Gemtuzumab } \\
\text { ozogamicin }\end{array}$ & Conventional chemotherapy alone & $0.95(0.83-1.08)$ & $\begin{array}{c}0.802 \\
(0.782-0.822)\end{array}$ \\
\hline Meta-analysis of Randomized Clinical Trials [50] & Idarubicin with cytarabine & Daunorubicin with cytarabine & $0.88(0.81-0.95)$ & \\
\hline Meta-analysis of Randomized Trials [51] & Autologous HSCT & Non- autologous HSCT & $1.05(0.91-1.21)$ & \\
\hline Systematic Review and Meta-analysis [52] & Allogeneic HSCT & Intensive or less intensive chemotherapy & $0.58(0.51-0.64)$ & \\
\hline Meta-Analysis of Retrospective Studies [53] & $\begin{array}{l}\text { Allogeneic HSCT with reduced-intensity } \\
\text { conditioning }\end{array}$ & $\begin{array}{l}\text { Allogeneic HSCT with myeloablative } \\
\text { conditioning }\end{array}$ & $0.97(0.88-1.07)$ & \\
\hline
\end{tabular}

Abbreviation: HSCT, hematopoietic stem cell transplantation; HR, hazard ratio; CI, confidence interval.

Figure. 4). The 5-year cancer-caused specific survival was $12.5 \%$ versus $23.6 \%$, respectively $(P<0.001)$.

Furthermore, we also evaluated the correlation between different factors and cancer-caused specific survival, using multivariate proportional hazard model by comparing matched datasets to unmatched complete datasets, as summarized in Table 7, 8. Various features were observed in all pairs of matched groups, and among them several independent prognostic factors emerged between matched unmarried versus married groups, including age, AML subtype and adjuvant therapy, and were similar to those in unmatched group. However, race (black, HR 0.946, 95\% CI: 0.898-0.998, $P=0.041$; other, HR $0.920,95 \%$ CI: $0.872-0.971, P=0.002$; unknown, HR $0.531,95 \%$ CI: $0.335-0.844, P=0.007$ ) was found to be associated with CSS in matched groups but not in unmatched. Similarly, sex was as a prognostic factor in unmatched groups but not in matched groups $(P>0.05)$. Among the never married versus married groups analysis, age ( $>55$ years, HR 1.611, 95\% CI: 1.529-1.698, $P<$ 0.001 ), race (black, HR 1.549, 95\% CI: 1.449-1.656, $P<$ 0.001 ), sex (male, HR 1.117, 95\% CI: 1.062-1.176, $P<$
0.001 ) and adjuvant therapy (radiation, HR $0.844,95 \%$ CI: $0.755-0.944, P=0.003$ ) were observed to be associated with CSS in matched groups, while in unmatched groups, some additional prognostic factors were also identified; age ( $>55$ years, HR 2.165, 95\% CI: 2.094-2.237, $P<$ 0.001 ), race (other, HR 1.053, 95\% CI: $1.002-1.106, P=$ 0.041 ), sex (male, HR 1.160, 95\% CI: 1.126-1.194, $P<$ 0.001 ), AML subtype (others, HR $0.733,95 \%$ CI: 0.712 $0.754, P<0.001$ ) and adjuvant therapy (radiation, HR $0.716,95 \%$ CI: $0.664-0.771, P<0.001)$. The age $>55$ years and diagnosis of AML, NOS eventually increased the risk of CSS in widowed and married group patients. Being male (HR 1.160, 95\% CI: 1.126-1.194, $P<0.001$ ) was associated with poor CSS in matched widowed versus married groups, while not in unmatched groups (HR $0.998,95 \%$ CI: $0.971-1.025, P=0.887)$. Also the similar result was observed in adjuvant therapy as receiving radiation (HR 0.725, 95\% CI: 0.672-0.783, $P<0.001$ ), which appeared as a favorable factor in unmatched group but did not reach statistical significance in matched group (HR 0.871, 95\% CI: $0.737-1.030, P=0.106$ ). 


\section{DISCUSSION}

In general, our study has been able to show that patients with unmarried status including those who were widowed or divorced have relatively greater risk of death due to primary AML, in comparison to patients who were married. In addition, we also observed that the specific association between marital status and survival outcomes was significant. Consistent with our data, similar conclusions have also been achieved in patients with solid tumors like, lung cancer, colorectal cancer, pancreatic cancer, liver cancer, esophagus cancer, head/neck cancer, non-Hodgkin lymphoma, thyroid cancer, gastric cancer and cervical cancer [7, 9, 11, 14, 22-25]. However, contrary to these observations, the study by Fintel AE [16] demonstrated that marital status did not influence the outcomes of acute lymphoblastic leukemia, while intrinsic differences in disease and disease-specific therapies did. Thus, we have directly compared the published HRs for the overall survival benefit of conventional chemotherapy and/or hematopoietic stem cell transplantation (HSCT) from meta-analysis and systematic reviews, and observed that HRs for the cancer-specific survival benefit correlated with marriage in this study (Table 9). The survival benefit associated with marriage was greater than the several published survival benefits of various treatments (high doses of daunorubicin, high dose of cytarabine, Gemtuzumab ozogamicin included chemotherapy, idarubicin with cytarabine, autologous HSCT and Allogeneic HSCT with reduced-intensity conditioning).

Importantly, we also observed some additional correlations in our study. Like, being never married was observed to be associated with favorable cancer-caused specific survival in general and in almost each subgroup adjusted by each prognostic variable in the univariate and log-rank analysis, when compared with married status. However, the multivariate analysis showed that never married AML patients actually had worse CSS in comparison to married status, and this might be attributed to the fact that population of being never married consisted of relatively more patients aged 15-55 years and were females. These 2 variables were later observed to be independent prognostic factors for survival. Thus, the result was adjusted when independent prognostic factors were integrated in the multivariate analysis. Our hypothesis was further validated when 1:1 matched groups were analyzed similarly using demographic and clinicopathological variables. The married patients showed remarkably better cancer-caused specific survival than never married patients in the log-rank test. The results from matched groups largely simulated the conditions of prospective study in the limited retrospective database, and were more persuasive than unmatched data [18]. Surprisingly, being male did not seem to influence the survival outcome in the matched married versus unmarried groups, while it actually increased the risk in unmatched groups. More interestingly, it even protected patients from AML-caused specific death in the matched widowed versus married groups. As this database included more males than females, thus it could be likely for males to display a little greater risk (HR 1.068) than females. As AML has not been a malignant tumors with significant gender differences [26], we do not regard sex as a remarkable prognostic factor in AML patients. Also it was noticed that when being widowed, male patients trends to act more optimistically towards disease and emerged with solid economic capability than female patients $[4,5,9]$. Hence, it was no surprise that male patients may survive a while longer in matched widowed versus married groups. Notably, it is quite common that unmarried adults usually live "with other persons" in modern society, which SEER database failed to record. So, we suggested that prognostic factors may differ in diverse marital status groups, and living with someone other than a spouse might not confer the similar protective benefit as marriage. Overall, our study emphasized about the substantial impact of marriage or more accurately social support on AML survival. Thus, it can be deduced from our study that providing social support to vulnerable populations such as single or widowed patients, could considerably increase the ratio of positive remission and survival.

Notably, socio-demographic factors have actually been shown to impact the disease outcome in multiple health conditions especially in countries and regions with limited access to free care [27, 28]. Marital status can have positive effect on AML diagnosis and subsequent treatment, as spouses can definitely advice patients to pay more medical attention for suspicious symptoms. Not only this, spouses can also play an important role in management of the disease [29]. Many studies have attempted to explain the reason for the correlation of married status with better survival in cancer by adjusting demographics, stage and treatment, and one reason which seems to be probable is that married patients adhere to the prescribed treatments better than unmarried [30-32]. There are potentially additional underlying etiologies which can explain the benefits of marriage on cancer-cause specific survival. Since the diagnosis of hematological malignancies usually results in more grieved outcome than other hematological diagnoses $[33,34]$, but it has been observed that married patients showed lower risk of major depression or anxiety than their unmarried counterparts [35], as emotional burden is shared by an intimate partner. Pessimism is another negative mediator between marital status and adherence to therapeutic approaches. Patients with depression generally undergo authoritative treatment less often and thus would display poor survival outcome [36-38]. With the change of marital status, the patient situation appears to become more complicated. It is generally expected that married patients may benefit in terms of emotional and social support in comparison to others who are widow, divorce or separate, as these 
patients definitely undergo more social and financial stress. As a result these patients cannot cope with stress and develop a negative attitude towards disease or even life, and should not be overlooked. It can be advised that physicians should screen unmarried AML patients, especially those who are experiencing marital upheaval and display pessimistic attitude. In addition they should be referred to mental health specialists, if typical symptoms are identified.

Many studies investigating the impact of marriage usually focus on patients with a single cancer just like the current study. However, there are some additional population-based studies which evaluated the impact of marriage on patient's outcomes in numerous cancers. The studies by Goodwin et al. [39] and Lai et al. [40] concluded that marital status has very limited effect on overall survival in cancer patients. The study by Aizer et al. [7] proposed a novel view about the significant correlation between marital status and cancer-specific mortality for many cancers including 10 leading malignancies, and their evaluation was based on 1,260,898 contemporary cancer patients throughout the United State. These results were consistent with our study and support our conclusions.

However, there were also few potential limitations of our study. First, our result could not be extended to AML patients from Asia, African, Latin America or even Europe. Second, our study lacked the data related to chemotherapy or HSCT. Third, some patients who cohabitated with a partner privately in the absence of marriage, were regarded as unmarried by SEER database, but these patients might survive longer than actually unmarried patients, hence has the tendency to bias our results. Finally, there was no information about the patients addiction to alcohol, smoke or maybe drug abuse in the SEER database, and these factors can also impact the survival of AML patients [41, 42]. The studies by Park $\mathrm{B}$ et al. and Balekang GB et al. have indicated that patients with unmarried status were more prone to such addiction habits $[43,44]$. So, the physician should pay attention to such adverse factors, particularly in unmarried AML patients. Nevertheless, despite these limitations, our study still highlighted the importance of social support as well as marital status, in significantly improving the therapeutic effects in unmarried AML patients.

Overall, our study demonstrated that unmarried AML patients are at a greater risk of cancer-specific mortality, and physicians should definitely evaluate the information about the social status/support of these patients, and when required should counsel and provide health resources targeting towards social support. This intervention may help to improve the rate of cancer-caused specific mortality in unmarried AML patients.

\section{CONFLICTS OF INTEREST}

The authors declare no conflict of interest.

\section{REFERENCES}

1. Dombret H, Gardin C. An update of current treatments for adult acute myeloid leukemia. Blood. 2016; 127:53-61.

2. Tabak I, Mazur J. Social support and family communication as factors protecting adolescents against multiple recurrent health complaints related to school stress. Developmental period medicine. 2016; 20:27-39.

3. Malhotra R, Ang SJ. Social Support for Older Adults-A Bane or a Boon for their Health? Ann Acad Med Singapore. 2016; 45:172-73.

4. Wang L, Wilson SE, Stewart DB, Hollenbeak CS. Marital status and colon cancer outcomes in US Surveillance, Epidemiology and End Results registries: does marriage affect cancer survival by gender and stage? Cancer Epidemiol. 2011; 35:417-22.

5. Li Q, Gan L, Liang L, Li X, Cai S. The influence of marital status on stage at diagnosis and survival of patients with colorectal cancer. Oncotarget. 2015; 6:7339-47. doi: 10.18632/oncotarget.3129.

6. Osborne C, Ostir GV, Du X, Peek MK, Goodwin JS. The influence of marital status on the stage at diagnosis, treatment, and survival of older women with breast cancer. Breast Cancer Res Treat. 2005; 93:41-47.

7. Aizer AA, Chen MH, McCarthy EP, Mendu ML, Koo S, Wilhite TJ, Graham PL, Choueiri TK, Hoffman KE, Martin $\mathrm{NE}, \mathrm{Hu}$ JC, Nguyen PL. Marital status and survival in patients with cancer. J Clin Oncol. 2013; 31:3869-76.

8. Tannenbaum SL, Zhao W, Koru-Sengul T, Miao F, Lee D, Byrne MM. Marital status and its effect on lung cancer survival. Springerplus. 2013; 2:504.

9. Shi RL, Qu N, Lu ZW, Liao T, Gao Y, Ji QH. The impact of marital status at diagnosis on cancer survival in patients with differentiated thyroid cancer. Cancer Med. 2016; 5:2145-54

10. Olsen MH, Bøje CR, Kjær TK, Steding-Jessen M, Johansen C, Overgaard J, Dalton SO. Socioeconomic position and stage at diagnosis of head and neck cancer - a nationwide study from DAHANCA. Acta Oncol. 2015; 54:759-66.

11. He XK, Lin ZH, Qian Y, Xia D, Jin P, Sun LM. Marital status and survival in patients with primary liver cancer. Oncotarget. 2016 Aug 5. doi: 10.18632/oncotarget.11066. [Epub ahead of print].

12. Hanske J, Meyer CP, Sammon JD, Choueiri TK, Menon M, Lipsitz SR, Noldus J, Nguyen PL, Sun M, Trinh QD. The influence of marital status on the use of breast, cervical, and colorectal cancer screening. Prev Med. 2016; 89:140-45.

13. Hurtado JL, Bacigalupe A, Calvo M, Esnaola S, Mendizabal N, Portillo I, Idigoras I, Millán E, Arana-Arri E. Social inequalities in a population based colorectal cancer 
screening programme in the Basque Country. BMC Public Health. 2015; 15:1021.

14. Jin JJ, Wang W, Dai FX, Long ZW, Cai H, Liu XW, Zhou Y, Huang H, Wang YN. Marital status and survival in patients with gastric cancer. Cancer Med. 2016; 5:1821-29.

15. Borate UM, Mineishi S, Costa LJ. Nonbiological factors affecting survival in younger patients with acute myeloid leukemia. Cancer. 2015; 121:3877-84.

16. Fintel AE, Jamy $\mathrm{O}$, Martin MG. Influence of insurance and marital status on outcomes of adolescents and young adults with acute lymphoblastic leukemia. Clin Lymphoma Myeloma Leuk. 2015; 15:364-67.

17. Ryerson AB, Eheman CR, Altekruse SF, Ward JW, Jemal A, Sherman RL, Henley SJ, Holtzman D, Lake A, Noone AM, Anderson RN, Ma J, Ly KN, et al. Annual Report to the Nation on the Status of Cancer, 1975-2012, featuring the increasing incidence of liver cancer. Cancer. 2016; 122:1312-37.

18. Nussbaum DP, Rushing CN, Lane WO, Cardona DM, Kirsch DG, Peterson BL, Blazer DG 3rd. Preoperative or postoperative radiotherapy versus surgery alone for retroperitoneal sarcoma: a case-control, propensity scorematched analysis of a nationwide clinical oncology database. Lancet Oncol. 2016; 17:966-75.

19. Wang Y, Huang J, Rong L, Wu P, Kang M, Zhang X, Lu Q, Fang Y. Impact of age on the survival of pediatric leukemia: an analysis of 15083 children in the SEER database. Oncotarget. 2016; 7:83767-74. doi: 10.18632/ oncotarget.11765.

20. Knoble NB, Alderfer MA, Hossain MJ. Socioeconomic status (SES) and childhood acute myeloid leukemia (AML) mortality risk: analysis of SEER data. Cancer Epidemiol. 2016; 44:101-08.

21. Chen Y, Yang T, Zheng X, Yang X, Zheng Z, Zheng J, Liu T, Hu J. The outcome and prognostic factors of 248 elderly patients with acute myeloid leukemia treated with standard-dose or low-intensity induction therapy. Medicine (Baltimore). 2016; 95:e4182.

22. Fernandez-Pineda I, Hudson MM, Pappo AS, Bishop MW, Klosky JL, Brinkman TM, Srivastava DK, Neel MD, Rao BN, Davidoff AM, Krull KR, Mulrooney DA, Robison LL, Ness KK. Long-term functional outcomes and quality of life in adult survivors of childhood extremity sarcomas: a report from the St. Jude Lifetime Cohort Study. J Cancer Surviv. 2016; 11:1-12.

23. Martínez ME, Anderson K, Murphy JD, Hurley S, Canchola AJ, Keegan TH, Cheng I, Clarke CA, Glaser SL, Gomez SL. Differences in marital status and mortality by race/ ethnicity and nativity among California cancer patients. Cancer. 2016; 122:1570-78.

24. Zhang J, Gan L, Wu Z, Yan S, Liu X, Guo W. The influence of marital status on the stage at diagnosis, treatment, and survival of adult patients with gastric cancer: a populationbased study. Oncotarget. 2016; 8:22385-22405. doi: 10.18632/oncotarget.7399.
25. El Ibrahimi S, Pinheiro PS. The effect of marriage on stage at diagnosis and survival in women with cervical cancer. Psychooncology. 2016; 26:704-710.

26. O'Donnell MR, Tallman MS, Abboud CN, Altman JK, Appelbaum FR, Arber DA, Attar E, Borate U, Coutre SE, Damon LE, Lancet J, Maness LJ, Marcucci G, et al. Acute myeloid leukemia, version 2.2013. J Natl Compr Canc Netw. 2013; 11:1047-55.

27. Jakobsen L, Niemann T, Thorsgaard N, Thuesen L, Lassen JF, Jensen LO, Thayssen P, Ravkilde J, Tilsted HH, Mehnert F, Johnsen SP. Dimensions of socioeconomic status and clinical outcome after primary percutaneous coronary intervention. Circ Cardiovasc Interv. 2012; 5:64148.

28. Vallgårda S. Addressing individual behaviours and living conditions: four Nordic public health policies. Scand J Public Health. 2011; 39:6-10.

29. Aizer AA, Paly JJ, Zietman AL, Nguyen PL, Beard CJ, Rao SK, Kaplan ID, Niemierko A, Hirsch MS, Wu CL, Olumi AF, Michaelson MD, D'Amico AV, Efstathiou JA. Multidisciplinary care and pursuit of active surveillance in low-risk prostate cancer. J Clin Oncol. 2012; 30:3071-76.

30. Costa AL, Heitkemper MM, Alencar GP, Damiani LP, Silva RM, Jarrett ME. Social Support Is a Predictor of Lower Stress and Higher Quality of Life and Resilience in Brazilian Patients With Colorectal Cancer. Cancer Nurs. 2016 May 11. [Epub ahead of print].

31. Iannarino NT, Scott AM, Shaunfield SL. Normative Social Support in Young Adult Cancer Survivors. Qual Health Res. 2016; 27:271-284.

32. Fong AJ, Scarapicchia TM, McDonough MH, Wrosch C, Sabiston CM. Changes in social support predict emotional well-being in breast cancer survivors. Psychooncology. 2016.

33. Albrecht TA, Boyiadzis M, Elswick RK Jr, Starkweather A, Rosenzweig M. Symptom Management and Psychosocial Needs of Adults With Acute Myeloid Leukemia During Induction Treatment: A Pilot Study. Cancer Nurs. 2016 Sep 14. [Epub ahead of print].

34. Ghodraty-Jabloo V, Alibhai SM, Breunis H, Puts MT. Keep your mind off negative things: coping with long-term effects of acute myeloid leukemia (AML). Supportive care in cancer. 2016; 24:2035-2045.

35. Ng DL, Leong YC, Gan GG. Quality of life amongst lymphoma survivors in a developing country. Supportive care in cancer. 2016; 24:5015-5023.

36. Charlson FJ, Baxter AJ, Dua T, Degenhardt L, Whiteford HA, Vos T. Excess Mortality from Mental, Neurological, and Substance Use Disorders in the Global Burden of Disease Study 2010. In: Patel V, Chisholm D, Dua T, Laxminarayan R, Medina-Mora ME, editors. Mental, Neurological, and Substance Use Disorders: Disease Control Priorities. Volume 4. 3rd ed. Washington (DC); 2016. 
37. Ghoneim MM, O'Hara MW. Depression and postoperative complications: an overview. BMC Surg. 2016; 16:5.

38. Polanski J, Jankowska-Polanska B, Rosinczuk J, Chabowski M, Szymanska-Chabowska A. Quality of life of patients with lung cancer. Onco Targets Ther. 2016; 9:1023-28.

39. Goodwin JS, Hunt WC, Key CR, Samet JM. The effect of marital status on stage, treatment, and survival of cancer patients. JAMA. 1987; 258:3125-30.

40. Lai H, Lai S, Krongrad A, Trapido E, Page JB, McCoy CB. The effect of marital status on survival in late-stage cancer patients: an analysis based on surveillance, epidemiology, and end results (SEER) data, in the United States. Int J Behav Med. 1999; 6:150-76.

41. Orsi L, Rudant J, Ajrouche R, Leverger G, Baruchel A, Nelken B, Pasquet M, Michel G, Bertrand Y, Ducassou S, Gandemer V, Lutz P, Saumet L, et al. Parental smoking, maternal alcohol, coffee and tea consumption during pregnancy, and childhood acute leukemia: the ESTELLE study. Cancer Causes Control. 2015; 26:1003-17.

42. Rota M, Porta L, Pelucchi C, Negri E, Bagnardi V, Bellocco R, Corrao G, Boffetta P, La Vecchia C. Alcohol drinking and risk of leukemia-a systematic review and meta-analysis of the dose-risk relation. Cancer Epidemiol. 2014; 38:33945.

43. Park B, Kim SI, Seo SS, Kang S, Park SY, Lim MC. Health Behaviors and Associated Sociodemographic Factors in Cervical Cancer Survivors Compared with Matched NonCancer Controls. PLoS One. 2016; 11:e0160682.

44. Balekang GB, Dintwa KF. A comparison of risky sexual behaviours between circumcised and uncircumcised men aged 30-44 years in Botswana. Afr Health Sci. 2016; $16: 105-15$.

45. Li D, Wang L, Zhu H, Dou L, Liu D, Fu L, Ma C, Ma X, Yao Y, Zhou L, Wang Q, Wang L, Zhao Y, et al. Efficacy of Allogeneic Hematopoietic Stem Cell Transplantation in Intermediate-Risk Acute Myeloid Leukemia Adult Patients in First Complete Remission: A Meta-Analysis of Prospective Studies. PLoS One. 2015; 10:e0132620.

46. Gong Q, Zhou L, Xu S, Li X, Zou Y, Chen J. High Doses of Daunorubicin during Induction Therapy of Newly Diagnosed Acute Myeloid Leukemia: A Systematic Review and Meta-Analysis of Prospective Clinical Trials. PLoS One. 2015; 10:e125612.
47. Li W, Gong X, Sun M, Zhao X, Gong B, Wei H, Mi Y, Wang J. High-dose cytarabine in acute myeloid leukemia treatment: a systematic review and meta-analysis. PLoS One. 2014; 9:e110153.

48. Li X, Xu SN, Qin DB, Tan Y, Gong Q, Chen JP. Effect of adding gemtuzumab ozogamicin to induction chemotherapy for newly diagnosed acute myeloid leukemia: a metaanalysis of prospective randomized phase III trials. Annals of oncology. 2014; 25:455-461.

49. Kharfan-Dabaja MA, Hamadani M, Reljic T, Pyngolil R, Komrokji RS, Lancet JE, Fernandez HF, Djulbegovic B, Kumar A. Gemtuzumab ozogamicin for treatment of newly diagnosed acute myeloid leukaemia: a systematic review and meta-analysis. Br J Haematol. 2013; 163:315-25.

50. Wang J, Yang YG, Zhou M, Xu JY, Zhang QG, Zhou RF, Chen B, Ouyang J. Meta-analysis of randomised clinical trials comparing idarubicin + cytarabine with daunorubicin + cytarabine as the induction chemotherapy in patients with newly diagnosed acute myeloid leukaemia. PLoS One. 2013; 8:e60699.

51. Wang J, Ouyang J, Zhou R, Chen B, Yang Y. Autologous hematopoietic stem cell transplantation for acute myeloid leukemia in first complete remission: a meta-analysis of randomized trials. Acta Haematol. 2010; 124:61-71.

52. Rashidi A, Ebadi M, Colditz GA, DiPersio JF. Outcomes of Allogeneic Stem Cell Transplantation in Elderly Patients with Acute Myeloid Leukemia: A Systematic Review and Meta-analysis. Biology of blood and marrow transplantation. 2016; 22:651-657.

53. Abdul Wahid SF, Ismail NA, Mohd-Idris MR, Jamaluddin FW, Tumian N, Sze-Wei EY, Muhammad N, Nai ML. Comparison of reduced-intensity and myeloablative conditioning regimens for allogeneic hematopoietic stem cell transplantation in patients with acute myeloid leukemia and acute lymphoblastic leukemia: a meta-analysis. Stem Cells Dev. 2014; 23:2535-52. 